\title{
CTRP12 Ameliorated Lipopolysaccharide-Induced Cardiomyocyte Injury
}

\author{
Meng-Qiao Zhou, ${ }^{a}$ E Jin, ${ }^{a}$ Jing Wu, ${ }^{b}$ Fei Ren, ${ }^{b}$ Yu-Zhi Yang, ${ }^{c}$ and Dong-Dong Duan*,c \\ ${ }^{a}$ Cardiology Function Examination Room, The First People's Hospital of Jingmen; 67 Xiangshan Avenue, Jingmen, \\ Hubei 448000, P. R. China: ${ }^{b}$ Department of Cardiology, The First People's Hospital of Jingmen; 67 Xiangshan \\ Avenue, Jingmen, Hubei 448000, P. R. China: and ${ }^{c}$ Department of Orthopaedic Surgery, The First People's Hospital \\ of Jingmen; 67 Xiangshan Avenue, Jingmen, Hubei 448000, P. R. China. \\ Received August 1, 2019; accepted November 24, 2019
}

C1q/tumor necrosis factor (TNF)-related protein 12 (CTRP12) is a secretory protein that participates in the regulation of glucose and lipid metabolism in obesity and diabetes. Its role in cardiovascular disease, particularly sepsis-induced cardiac injury, is unclear. Here, we stimulated cardiomyocytes with lipopolysaccharide (LPS) to establish an in vitro cardiomyocyte injury model and CTRP12 was overexpressed with an adenovirus delivery system. Overexpression of CTRP12 reduced the transcription and release of pro-inflammatory cytokines from LPS-stimulated cardiomyocytes, including TNF $\alpha$, interleukin-1 (IL-1), and IL-6. Reactive oxygen species (ROS) level increased and the oxidation/redox system was disturbed in LPS-stimulated cardiomyocytes, as evident from the decrease in superoxide dismutase activity and an increase in reduced nicotinamide adenine dinucleotide phosphate (NADPH) oxidase activity and malondialdehyde level. CTRP12 overexpression decreased the increasing level of ROS and ameliorated the unbalance in the oxidation/redox system in LPS-stimulated cardiomyocytes. The viability of cardiomyocytes decreased after LPS stimulation, and the cells underwent apoptosis. CTRP12-overexpressing cardiomyocytes showed a decrease in the number of terminal deoxynucleotidyl transferase-mediated deoxyuridine triphosphate nick-end labeling (TUNEL)positive cells, and the ratio of $\mathrm{B}$ cell lymphoma $(\mathrm{Bcl})-1 / \mathrm{Bax}$ in these cells was recovered. In comparison with the control group, LPS-stimulated cardiomyocytes showed reduced expression of nuclear factor E2-related factor 2 (NRF2), while CTRP12-overexpressing cardiomyocytes showed elevated NRF2 expression. Smallinterfering RNA-mediated silencing of NRF2 expression in cardiomyocytes resulted in the inhibition of the protective effects of CTRP12. Thus, CTRP12 ameliorated injury in LPS-stimulated cardiomyocytes in an NRF2-dependent manner.

Key words $\mathrm{Clq} /$ tumor necrosis factor-related protein 12; lipopolysaccharide; oxidative stress; nuclear factor E2-related factor 2

\section{Introduction}

Sepsis is a medical emergency and demands immediate treatment. ${ }^{1)}$ The mortality rate associated with sepsis is surprisingly high worldwide and ranges from 10 to $50 \%$ in diagnosed patients. Organ injury and septic shock lead to high mortality rates. ${ }^{2}$ ) Although standard treatment options to control bacterial growth and inflammation during the early onset of sepsis are available, patients frequently develop secondary complications that lead to severe sepsis., ${ }^{3,4)}$ Cardiac dysfunction, a major cause of multi-organ failure, is a common consequence of severe sepsis. ${ }^{5}$ More than $60 \%$ of ICU patients diagnosed with sepsis show clinical manifestations in the form of cardiac dysfunction, and a mortality rate of 70 to $90 \%$ is reported. ${ }^{6}$ The bacterial endotoxin lipopolysaccharide (LPS) is the principle component involved in multi-organ failure following septic shock. ${ }^{7)}$ LPS causes initial hyperinflammatory response through an increase in the production of tumor necrosis factor alpha (TNF $\alpha$ ), interleukin (IL)- $1 \beta$, IL-6, and chemokines. ${ }^{4}$ These hyper-inflammatory factors induce the progression of arrhythmia, myocardial ischemia and infarction, and heart failure and profoundly change cardiac structure, electrical activity, and pump function. ${ }^{8,9)}$ Although tremendous efforts have been directed to study the molecular mechanism underlying the pathogenesis of sepsis-induced cardiac dysfunction, the information needed to target sepsisinduced cardiac dysfunction is sparse.
The adipokine C1q/TNF-related protein 12 (CTRP12; adipolin) plays an important role in insulin sensitivity and fighting inflammation. ${ }^{10)}$ Lower circulating levels of CTRP12 have been observed in rodent models of obesity and diabetes. ${ }^{10)}$ CTRP12 protein is known to regulate glucose ${ }^{11)}$ and lipid metabolism, ${ }^{12)}$ and patients with coronary artery disease show decreasing serum CTRP12 levels. ${ }^{13)}$ These observations indicate the potential role of CTRP12 in sepsis-induced cardiac injury. Here, we evaluate the effect of CTRP12 on LPS-stimulated cardiomyocytes and investigate the underlying mechanism.

\section{Experimental}

H9c2 Cardiomyocyte Culture The H9c2 cardiomyocyte line was obtained from the Cell Bank of the Chinese Academy of Sciences (Shanghai, China) and used after efficient recovery. Cells were cultured in Dulbecco's modified Eagle's medium (DMEM; C11995, GIBCO, Thermo Fisher Scientific, U.S.A.) supplemented with $10 \%$ fetal bovine serum (FBS) ${ }^{7)}$ To overexpress CTRP12, the full-length rat CTRP12 cDNA under the control of the cytomegalovirus promoter was inserted into replication-defective adenoviral vectors (adenoviral CTRP12). An adenoviral vector encoding the $L a c Z$ gene was used as a control (adenoviral LacZ protein) (HAN biology Tech, Shanghai, China). Cells were transfected with adenovirus (Ad)-CTRP12 (multiplicity of infection $=100$ ) to overexpress CTRP12 8h before LPS stimulation in FBS-free 
DMEM. The cells were treated with LPS (L2630, SigmaAldrich, U.S.A.) for another $24 \mathrm{~h}$.

Neonatal Rat Cardiomyocyte (NRCM) Isolation and Culture The primary cultures of cardiomyocytes were prepared as previously described. ${ }^{14)}$ Cells from the heart of 1- to 7-d-old Sprague-Dawley rats were seeded at a density of $1 \times 10^{6}$ cells/well in a six-well plate in DMEM/F12 medium (A4192002, Gibco-BRL) supplemented with 10\% FBS (GibcoBRL, 10099-133) and $100 \mathrm{U} / \mathrm{mL}$ penicillin and $100 \mathrm{mg} / \mathrm{mL}$ streptomycin (Gibco-BRL, 15140). After 48h, the medium was replaced with DMEM/F12 medium supplemented with $0.2 \%$ FBS and 0.1 mM 5-bromo-2-deoxyuridine (BrdU, Sigma, B5002). Cells were transfected with Ad-CTRP12 to overexpress CTRP12 $8 \mathrm{~h}$ before LPS stimulation in FBS-free DMEM. The cells were eventually treated with LPS for another $24 \mathrm{~h}$.

Cell Viability Assay Cell Counting Kit-8 (CCK-8; SigmaAldrich, 03285) assay was used to detect cellular viability, as previously described. ${ }^{7)}$ Briefly, cells were incubated with $10 \mu \mathrm{L}$ diluted CCK- 8 solution after treatment for $4 \mathrm{~h}$. The absorbance was measured using an enzyme-linked immunosorbent assay (ELISA) plate reader at $450 \mathrm{~nm}$ wavelength (Synergy HT, Bio-tek, Vermont, U.S.A.). Cell viability was calculated as the percentage viable cells as compared with the control group.

Oxidative Stress Reactive oxygen species (ROS) level was detected using dichlorofluorescein diacetate assay (S0033, DCFH-DA, Beyotime Biotechnology, Shanghai, China). Briefly, after treatment, cells were labeled with $10 \mu \mathrm{M}$ DCFH-DA for $60 \mathrm{~min}$ at $37^{\circ} \mathrm{C}$. A fluorescence microplate reader (excitation wavelength/emission wavelength: $485 / 525 \mathrm{~nm}$ ) and light microscope (BX51TRF; Olympus Corporation, Tokyo, Japan) were used for the detection of immunofluorescence. Superoxide dismutase (SOD) activity (S0101, Beyotime Biotechnology), reduced nicotinamide adenine dinucleotide phosphate (NADPH) oxidase activity (S0179, Beyotime Biotechnology), and malondialdehyde (MDA, S0131, Beyotime Biotechnology) production were evaluated as per the manufacturer's instruction (Beyotime Biotechnology).

Terminal Deoxynucleotidyl Transferase dUTP Nick End Labeling (TUNEL) Staining TUNEL staining was performed as previously described ${ }^{7)}$ using a commercial regent (EMD Millipore, 4500-0121). Nucleus was stained with 4',6-diamidino-2-phenylindole (DAPI; Invitrogen Life Technologies). TUNEL-positive cells were counted under a microscope (BX51; Olympus Corp.) and expressed as the percentage of apoptotic nuclei/total nuclei.

Western Blot Analysis After treatment, cytosolic and nuclear proteins were extracted using Nuclear and Cytoplasmic Protein Extraction Kit (P0027, Beyotime Biotechnology). Total proteins were extracted after lysing cells with radioimmunoprecipitation assay (RIPA) lysis buffer. The proteins were separated on $10 \%$ sodium dodecyl sulfate polyacrylamide gel electrophoresis (SDS-PAGE) gels, and the separated bands were transferred onto polyvinylidene difluoride membranes. The membranes were incubated with the following primary
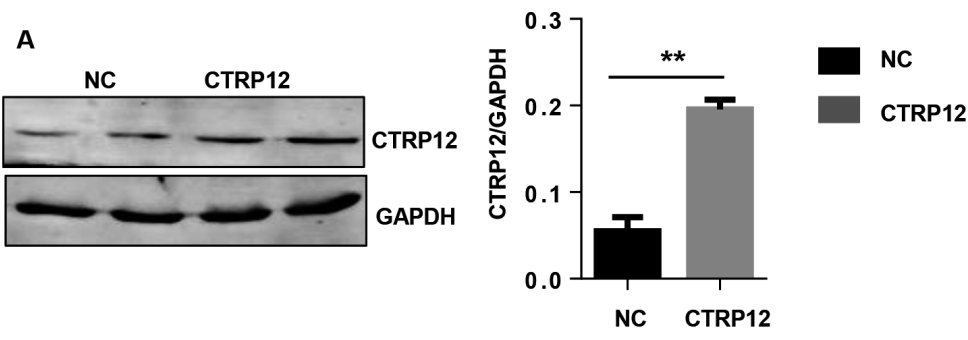

B
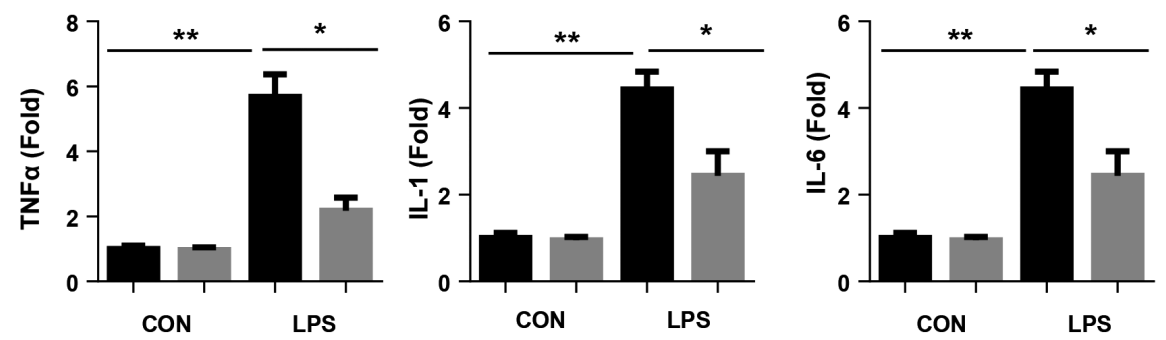

C
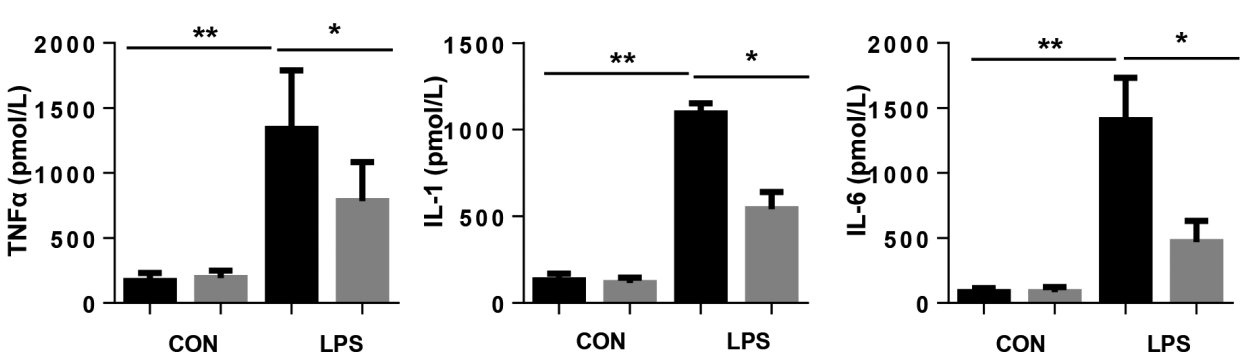

Fig. 1. CTRP12 Reduces the Hyper-Inflammatory Response Induced by LPS

A. H9c2 cells were transfected with Ad-CTRP12. Expression of CTRP12 in cells transfected with Ad-CTRP12 ( $n=4)$. B-C. H9c2 cells were transfected with AdCTRP12 and treated with LPS $(10 \mu \mathrm{g} / \mathrm{mL})$ for $24 \mathrm{~h}$. B. Transcription level of TNF $\alpha$, IL-1, and IL-6 in H9c2 cells $(n=6)$. C. Release of TNF $\alpha$, IL-1, and IL-6 from H9c2 cells $(n=6)$. $* p<0.05 ; * * p<0.01$. 

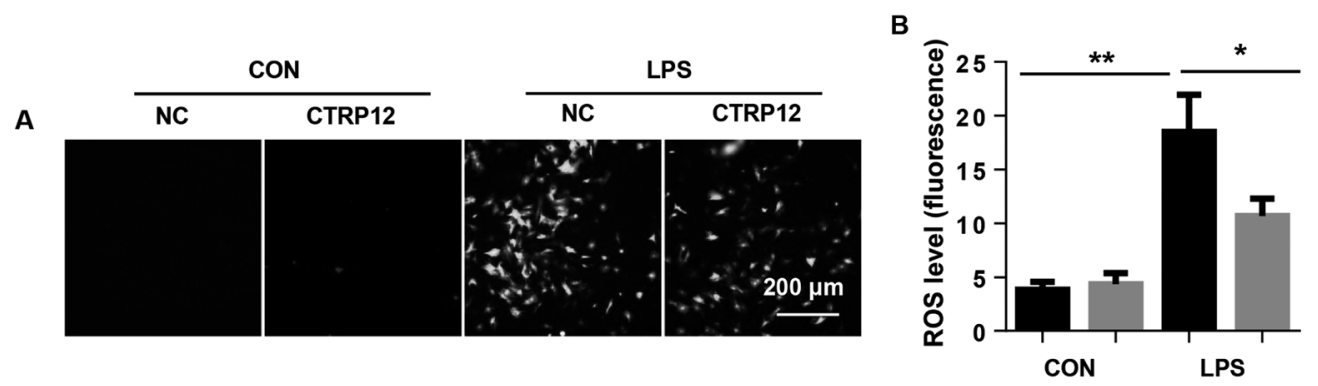

C
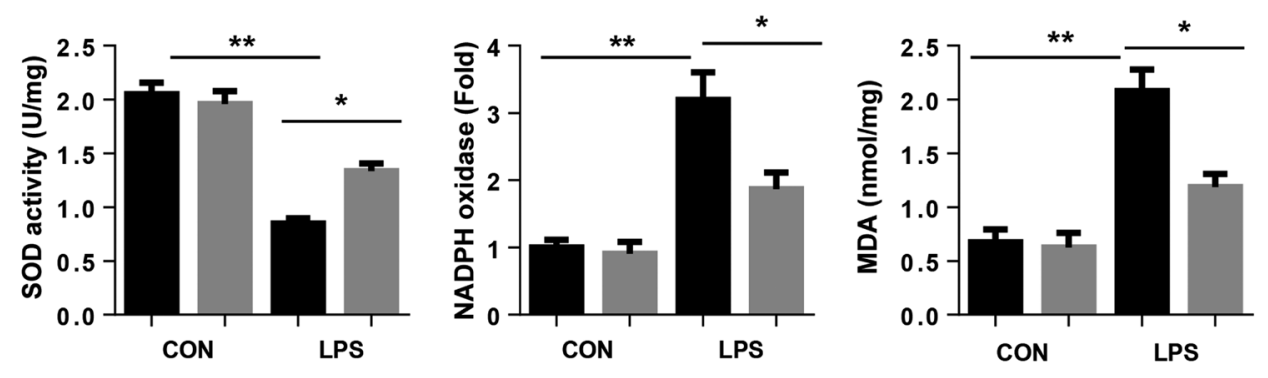

D
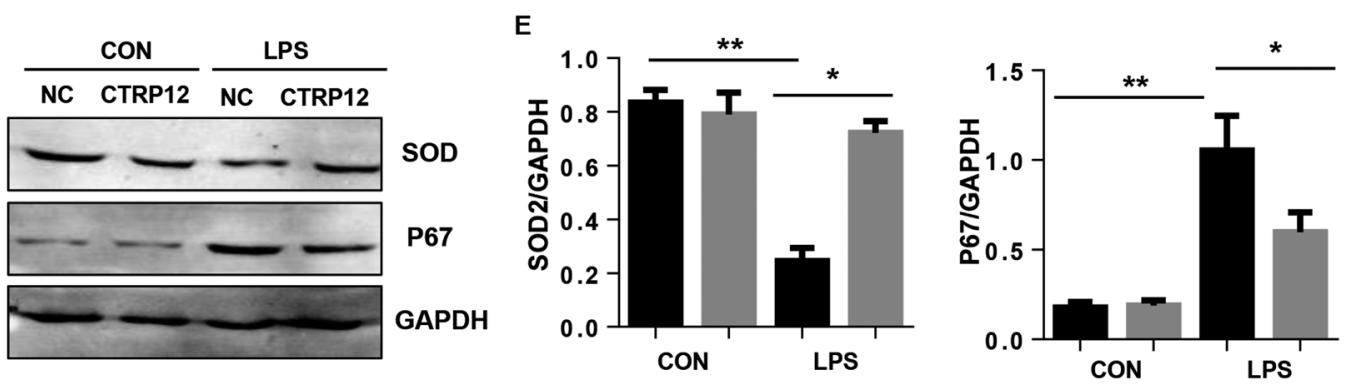

Fig. 2. CTRP12 Ameliorates the Excessive Oxidative Stress Induced by LPS

H9c2 cells were transfected with Ad-CTRP12 and treated with LPS $(10 \mu \mathrm{g} / \mathrm{mL})$ for $24 \mathrm{~h}$. A and B. ROS level in H9c2 cells $(n=6)$ as detected by light microscopy (A) and fluorescence microplate reader (B). C. SOD activity, NADPH oxidase activity, and MDA level in H9c2 cells $(n=6)$. D and E. Expression levels of SOD2 and P67 in H9c2 cells $(n=4) . * p<0.05 ; * * p<0.01$

A

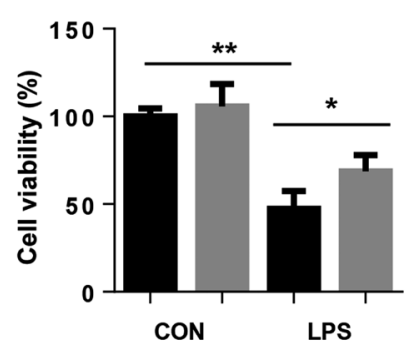

C

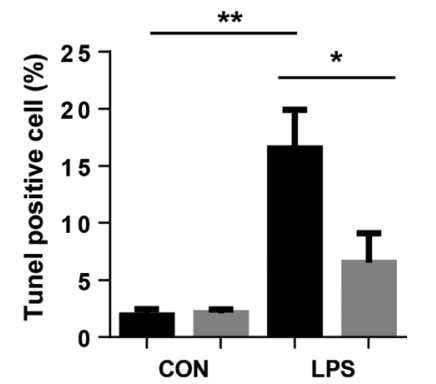

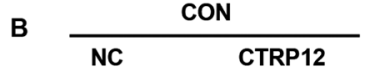
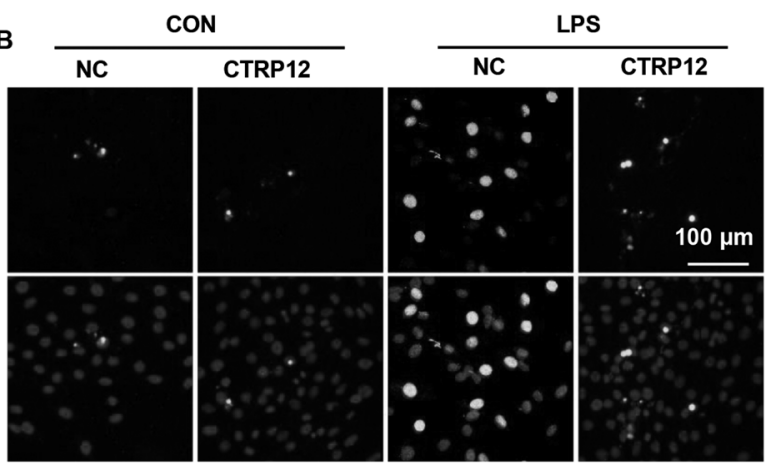

D
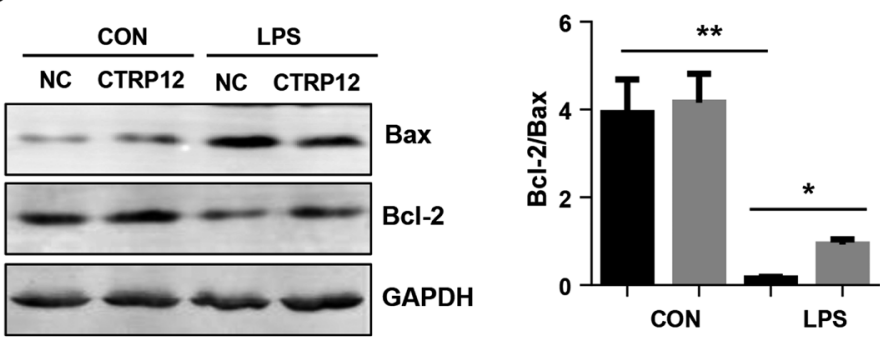

Fig. 3. CTRP12 Ameliorates Cell Apoptosis Induced by LPS

H9c2 cells were transfected with Ad-CTRP12 and treated with LPS $(10 \mu \mathrm{g} / \mathrm{mL})$ for $24 \mathrm{~h}$. A. Viability of H9c2 cells was detected with the CCK-8 assay $(n=5)$. B and C. TUNEL staining in H9c2 cells $(n=5)$. D. Expression level of Bcl-2 and Bax in H9c2 cells $(n=4) . *^{*} p<0.05 ; * * p<0.01$. 
antibodies overnight at $4^{\circ} \mathrm{C}$ : $\mathrm{Bax}(\mathrm{ab} 182733)$, B cell lymphoma (Bcl)-2 (ab196495), SOD2 (ab13533), P67 (ab134124), heme oxygenase-1 (HO-1; ab13248), peroxiredoxin-1 (Prx-1; ab205718), nuclear factor E2-related factor 2 (NRF2; ab137550), Lamin B (ab16048), and glyceraldehyde 3-phosphate dehydrogenase (GAPDH; ab181602) (all purchased from Abcam and used at $1: 1000$ dilution). The membranes were eventually probed with secondary antibodies (926-32211/926-32210, LI-COR Biosciences), and a two-color infrared imaging system was used to scan membranes and obtain target bands (Odyssey, LI-COR). GAPDH was used as a reference.

Real-Time Quantitative PCR (RT-qPCR) After extraction with TRIzol, the RNA was reversed transcribed into cDNA with oligo(dT) primers (04896866001, Roche Diagnostics, Basel, Switzerland) using a Transcriptor First Strand cDNA Synthesis kit (04896866001; Roche Diagnostics). Quantification of PCR amplification was carried out with LightCycler 480 SYBR $^{\circledR}$ Green 1 Master Mix (04707516001; Roche Diagnostics) on a LightCycler ${ }^{\circledR} 480$ Real-time quantitative PCR System (Roche Diagnostics). GAPDH was used as an internal reference. The primers used were as follows: TNF $\alpha$ forward 3'-AGCATGATC CGA GAT GTGGAA-5' and reverse 3'-TAGACA GAA GAGCGTGGTGGC-5'; IL-1 $\beta$ forward $3^{\prime}$ GGGATGATGACGACC TGC TAG-5' and reverse $3^{\prime}$-ACC ACT TGT TGGCTT ATG TTC TG-5'; IL-6 forward 3'-GTT GCC TTC TTGGGACTGATG-5' and reverse $3{ }^{\prime}$-ATACTG GTC TGT TGT GGG TGG T-5'.

Immunofluorescence Staining The cells were washed thrice with phosphate-buffered saline (PBS), fixed with 4\% paraformaldehyde, and permeabilized with $0.1 \%$ Triton X-100 (Amresco, Cochran Road Solon, U.S.A.). The cells were incubated with the target primary antibody (anti-NRF2, Abcam, $1: 100$ dilution) for overnight, followed by treatment with Alexa Fluor 568 goat anti-rabbit IgG secondary antibody (A-11011, Invitrogen; Thermo Fisher Scientific, Inc.). SlowFade Gold antifade reagent with DAPI (S36939, Invitrogen; Thermo Fisher Scientific, Inc.) was used to stain cell nucleus. Quantification was performed with Image Pro-Plus 6.0.

Statistical Analysis All values are shown as the mean \pm standard deviation. Differences among the groups were determined with one-way ANOVA, followed by Tukey's post-hoc test. Unpaired Student's $t$-test was used for comparison between two groups. A value of $p<0.05$ was considered statistically significant.

\section{Results}

CTRP12 Reduces the Hyper-Inflammatory Response Induced by LPS We used an adenovirus system to overexpress CTRP12. As shown in Fig. 1A, the expression level of CTRP12 was higher in Ad-CTRP12-treated group than in the negative control group. We detected the initial inflammatory response induced by LPS stimulation. As shown in Fig. 1B, the transcription of pro-inflammatory cytokines TNF $\alpha$, IL-1, and IL-6 was higher in LPS-stimulated cells than in control cells, while these pro-inflammatory cytokine levels decreased in CTRP12-overexpressing cells after LPS stimulation. Moreover, we evaluated the release of these pro-inflammatory cytokines in the cell culture medium and found that TNF $\alpha$, IL-1, and IL-6 levels were higher in LPS-stimulated cells than in control cells. The levels of these pro-inflammatory factors decreased in CTRP12-overexpressing cells after LPS stimulation as compared with the negative control cells (Fig. 1C). These observations indicate that CTRP12 may relieve the initial inflammatory response induced by LPS stimulation.

CTRP12 Reduces the Oxidative Stress Induced by LPS Excessive oxidative stress damages cellular proteins, lipids, and DNA, ultimately leading to cell death. Thus, we detected the balance in the oxidation/redox system. As shown in Figs. $2 \mathrm{~A}$ and $\mathrm{B}$, the ROS levels were higher in LPS-stimulated cells than in control cells. In comparison with the negative control group, CTRP12-overexpressing cells showed a decrease in ROS level after LPS stimulation. The level of lipid peroxidation intermediate metabolite, MDA, increased in LPS group but decreased in CTRP12-overexpressing cells (Fig. 2C). We detected the activity of oxidase and anti-oxidase (Fig. 2B) after LPS stimulation and found that SOD activity decreased in the negative control group but increased in CTRP12-overexpressing cells. While the activity of NADPH oxidase increased in the negative control cells, it decreased in CTRP12-overexpressing cells (Fig. 2C). To explore whether CTRP12 regulates oxidative stress, we detected the protein levels of SOD2 and NADPH P67 phox (Fig. 2D) in response to LPS stimulation and found that the expression level of SOD2 reduced but that of P67 increased. CTRP12 overexpres-
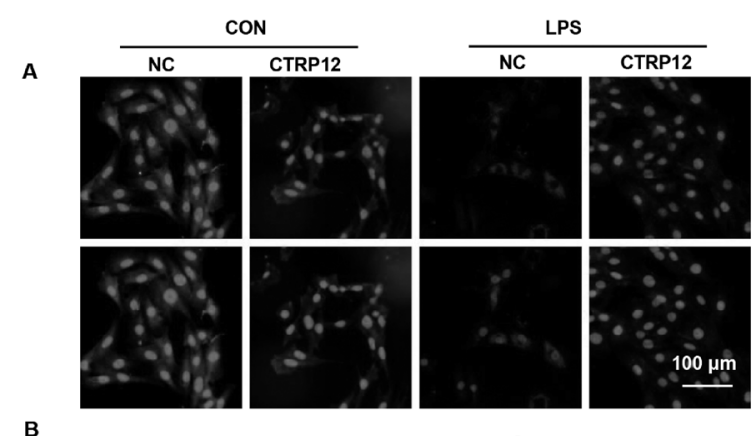

B
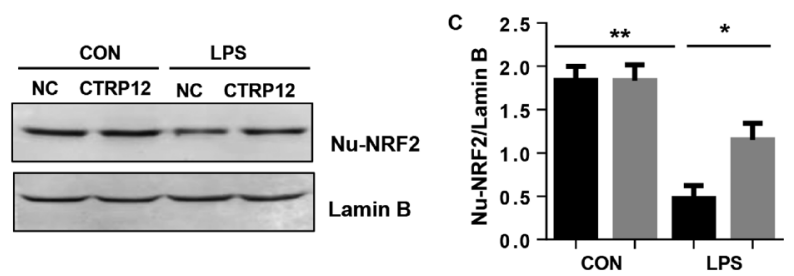

D
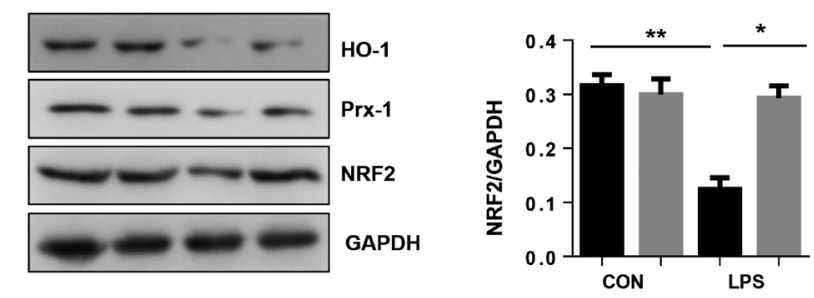

E
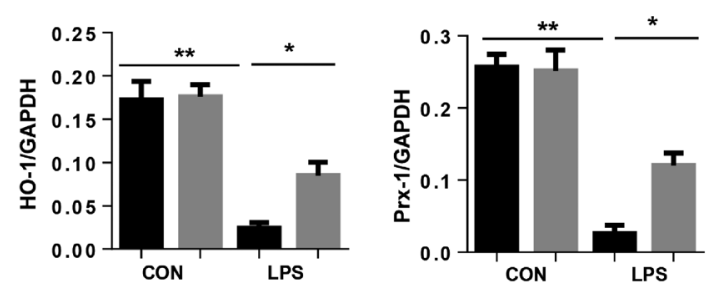

Fig. 4. CTRP12 Functions through NRF2

H9c2 cells were transfected with Ad-CTRP12 and treated with LPS $(10 \mu \mathrm{g} / \mathrm{mL})$ for $24 \mathrm{~h}$. A. NRF2 immunofluorescence staining for H9c2 cells $(n=5)$. B and C. Nuclear expression of NRF2 in H9c2 cells $(n=4)$. D and E. Protein levels of HO-1, Prx-1, and NRF2 in the cytoplasm of H9c2 cells $(n=4) .{ }^{*} p<0.05 ; * * p<0.01$. 


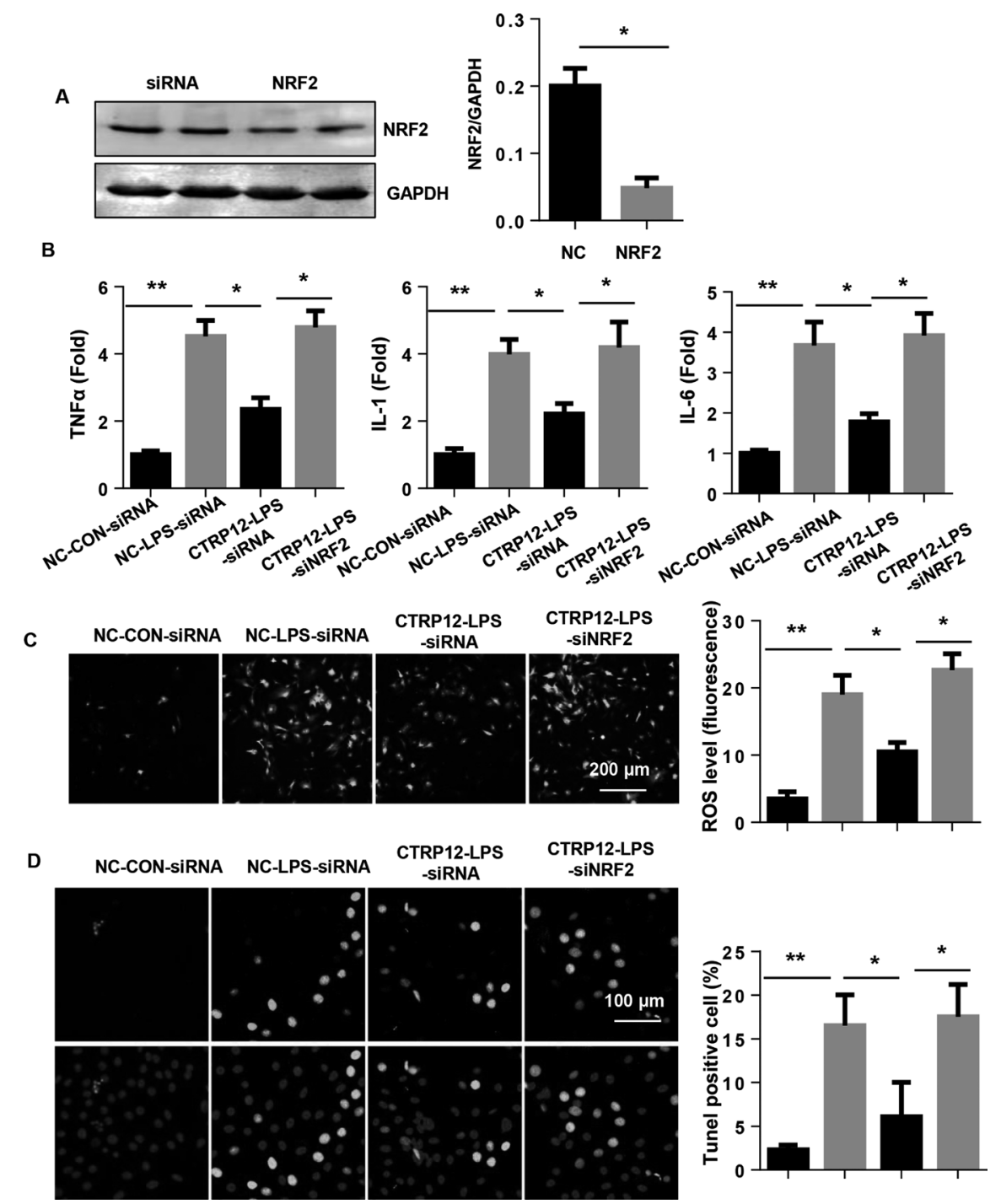

Fig. 5. NRF2 Silencing Reverses the Functional Role of CTRP12

A. H9c2 cells were transfected with NRF2 siRNA. Expression level of NRF2 after the transfection of cells with NRF2 siRNA $(n=4)$. B-D. H9c2 cells were transfected with NRF2 siRNA and Ad-CTRP12. B. Transcription levels of TNF $\alpha$, IL-1, and IL-6 in H9c2 cells $(n=6)$. C. ROS level in H9c2 cells $(n=6)$. D. TUNEL staining in H9c2 cells $(n=5) . * p<0.05 ; * p<0.01$.

sion increased SOD2 level and reduced P67 expression level. These data confirm that CTRP12 ameliorates oxidative stress by regulating the activity of both SOD and NADPH oxidase.

CTRP12 Ameliorates LPS-Induced Cell Apoptosis Programmed cell death is the key feature of sepsis-induced cardiomyopathy. We investigated whether the protective effect of CRTP12 on initial inflammation and oxidative stress could reduce the apoptosis of LPS-stimulated cardiomyocytes. As shown in Fig. 3A, cellular viability significantly decreased after LPS stimulation, while CTRP12 overexpression in LPSstimulated cardiomyocytes resulted in an increase in viability. We detected apoptosis by TUNEL staining. As shown in Figs. $3 \mathrm{~B}$ and $\mathrm{C}$, the number of TUNEL-positive cells dramatically increased in LPS-stimulated cells but decreased in CTRP12overexpressing cells. The expression ratio of the anti-apoptosis protein $\mathrm{Bcl}-2$ to pro-apoptosis protein $\mathrm{Bax}$ was measured by Western blotting. As a result, we found that Bcl-2/Bax ratio decreased in LPS-stimulated cells but was recovered in CTRP12-overexpressing cells.

CTRP12 Functions via NRF2 We investigated the mechanism underlying the protective effect of CTRP12. NRF2 is a transcriptional factor that mainly regulates the oxidation/ redox system. We observed reduction in the nuclear translocation of NRF2 in LPS-stimulated cells as compared with control cells (Fig. 4A). However, the nuclear translocation of NRF2 increased in CTRP12-overexpressing cells as compared with that in negative control cells. We also detected nuclear NRF2 expression and found it to be decreased in negative control cells but increased in CTRP12-overexpressing cells in response to LPS stimulation (Figs. 4B, C). The expression of NRF2 target genes, including $H O-1$ and $\operatorname{Pr} x-1$, was analyzed and found to be increased after CTRP12 overexpression in LPS-stimulated cells (Figs. 4D, E).

NRF2 Silencing Reverses the Functional Role of CTRP12 To explore whether NRF2 silencing would counteract the effect of CTRP12, we used an NRF2 small-interfering RNA (siRNA) to silence NRF2 expression in cardiomyocytes. The cells transfected with NRF2 siRNA showed a dramatic decrease in the level of NRF2 (Fig. 5A). The transcription of the inflammatory cytokines, including TNF $\alpha$, IL-1, and IL-6, 


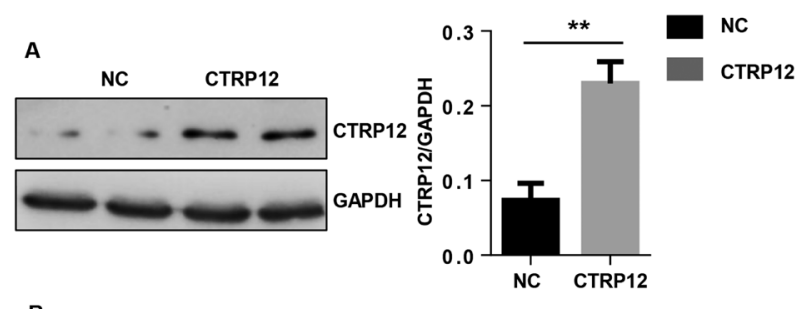

B
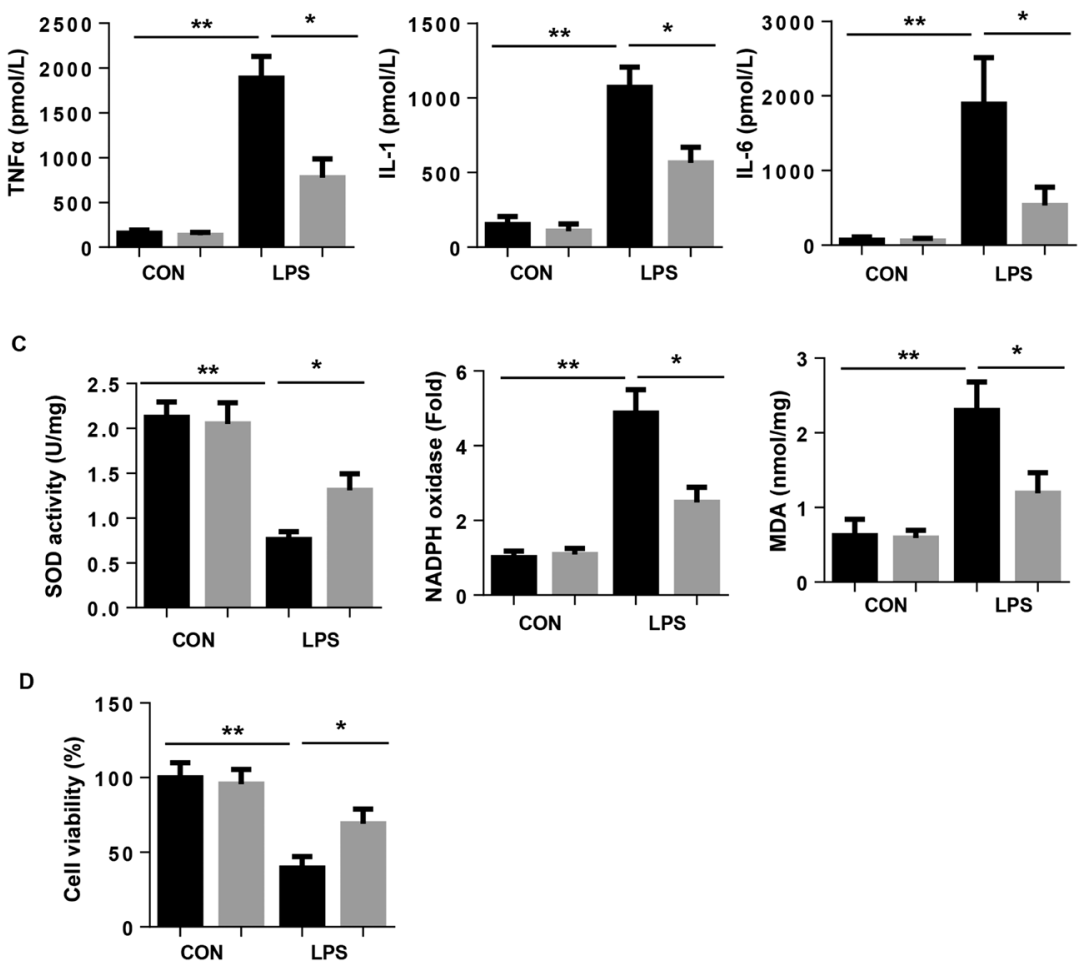

Fig. 6. CTRP12 Attenuates LPS-Induced Hyper-Inflammation and Oxidative Stress in NRCMs

A. NRCMs were transfected with Ad-CTRP12. The expression of CTRP12 was evaluated in cells transfected with Ad-CTRP12 $(n=4)$. B-D. NRCMs were transfected with Ad-CTRP12 and treated with LPS $(10 \mu \mathrm{g} / \mathrm{mL})$ for $24 \mathrm{~h}$. B. Release of TNF $\alpha$, IL-1, and IL-6 $(n=6)$. C. SOD activity, NADPH oxidase activity, and MDA level $(n=6)$. D. Cell viability was analyzed with the CCK-8 assay $(n=5)$. $* p<0.05 ; * *<0.01$.

increased in the cells from LPS group but decreased in the cells overexpressing CTRP12. A sharp increase in the levels of these factors was observed in the cells from NRF2 silencing group (Fig. 5B). Consistent with this result, we found that ROS level and TUNEL-positive cell number also increased in LPS group but decreased in CTRP12 overexpression group. However, a sharp increase in ROS level and TUNEL-positive cell number was observed in the cells from NRF2 silencing group (Figs. 5C, D). These data indicate that NRF2 silencing could counteract the protective effects of CTRP12.

CTRP12 Attenuates LPS-Induced Hyper-Inflammation and Oxidative Stress in NRCMs To confirm the functional role of CTRP12 in neonatal cardiomyocytes, NRCMs were isolated, cultured, and transfected with Ad-CTRP12 to overexpress CTRP12 (Fig. 6A). Consistent with the result observed with H9c2 cells, CTRP12 expression attenuated the LPS-induced inflammation and oxidative stress and preserved cellular viability (Figs. 6B-D).

\section{Discussion}

Sepsis is normally caused from bacterial infection, and the heart is the main target organ in serve septic shock. ${ }^{9)}$ The pathogenesis of sepsis-induced cardiomyopathy is associ- ated with an imbalance in inflammatory mediators, oxidative stress, mitochondrial dysfunction, abnormal calcium regulation, and endothelial dysfunction, all of which result in an irreversible damage to the heart. ${ }^{15)}$ Treatment for sepsis-induced cardiomyopathy is limited, thereby leading to an increase in mortality rate. ${ }^{15)}$ Here, we found that CTRP12 could reduce the initial inflammatory response induced by LPS and suppress the excessive oxidative stress and apoptosis in cardiomyocytes induced following LPS stimulation.

The lysis of Gram-negative bacteria results in the release of endotoxins, ${ }^{16)}$ which could reduce left ventricular ejection fraction (LVEF) and LV performance in healthy individuals. ${ }^{16)}$ Endotoxins are pathogen-associated molecular patterns that activate the receptors of not only the immune cells but also cardiomyocytes, leading to an inflammatory cascade and induction of the expression and release of inflammatory mediators. ${ }^{17)}$ The dominant inflammatory mediators that play a role in sepsis-induced cardiomyopathy are TNF $\alpha$, IL-1, and IL-6. ${ }^{15)}$ TNF $\alpha$ was shown to cause a dose-dependent decrease in LVEF in a septic shock model. ${ }^{18)}$ IL-1 cooperated with $\mathrm{TNF} \alpha$ to enhance the decrease in myocardial contractility. ${ }^{17)}$ IL-6 exerted negative inotropic effects on sepsis-induced cardiomyopathy. ${ }^{17)}$ CTRP12, an adipokine and a paralog of 
adiponectin, is a newly discovered molecule ${ }^{10)}$ known to show an independent connection with the risk of coronary artery disease (CAD). In patients with CAD, CTRP12 expression inversely correlated with homeostasis model assessment of insulin resistance (HOMA-IR), TNF $\alpha$, and IL-6. ${ }^{13)}$ These studies indicate the potential anti-inflammatory effects of CTRP12. In our study, we first investigated the direct effect of CTRP12 on cardiomyocytes in response to LPS stimulation. CTRP12 suppressed the expression and release of these key inflammatory mediators, thereby attenuating the initial inflammatory response and subsequently ameliorating cell damage.

Sepsis is characterized with an increase in the production of free radicals that are released from the cardiomyocyte mitochondria, eventually impairing the heart function. ${ }^{19)}$ The inability of the antioxidant protection mechanism to counteract the excessive ROS production may lead to the development of oxidative stress, thereby damaging cellular proteins, lipid, and DNA. ${ }^{19)}$ In cardiomyocytes, endotoxins induce the production of ROS through xanthine oxidase, NADH/NADPH oxidases, and mitochondria. ${ }^{20)}$ The increase in ROS level, in turn, causes mitochondria dysfunction and further induces ROS production and dysfunction of oxidative phosphorylation, thereby reducing ATP levels. ${ }^{19)}$ In response to irreversible mitochondrial dysfunction, the ratio of Bcl-2 to Bax is reversed, subsequently triggering cell death and organ dysfunctions. ${ }^{21)}$ In our study, we observed a decrease in ROS level and a re-balance in the oxidation/redox system in CTRP12-overexpressing cells. These phenomena ameliorated oxidative stress and may have contributed to the repair of the oxidation/redox system.

NRF2 as a transcriptional factor functions by binding to antioxidant response elements (AREs), the DNA sequences that respond to dietary antioxidants. ${ }^{22)}$ For instance, NQO1, HO-1, glutathione $S$-transferases, and glutamate-cysteine ligase catalytic and regulatory subunits are all considered as classic genes. $^{23)}$ Upon cleavage from the cytoplasmic protein Keap1, NRF2 translocates into the nucleus and binds to cis-acting AREs, thereby activating the transcription of antioxidants. ${ }^{22)}$ No study has reported the regulatory role of CTRP12 in NRF2 expression and activation. We found that the nuclear expression and translocation of NRF2 was elevated upon CTRP12 overexpression under LPS stimulation conditions. However, under physiological state, the expression and nuclear translocation of NRF2 was unaffected by CTRP12 overexpression. The expression and nuclear translocation of NRF2 may be driven by other factors, which cooperate with CTRP12 under pathological condition. Further studies are warranted to clarify the specific mechanism underlying the CTRP12-mediated regulation of NRF2.

In summary, CTRP12 suppressed the initial inflammatory response in cardiomyocytes after exposure to LPS. CTRP12 also reduced the subsequent oxidative stress and cellular apoptosis by enhancing the activation of NRF2. Thus, our study highlights a possible strategy for the treatment of sepsisinduced cardiomyopathy using CTRP12.

Acknowledgments This study was supported by General Science and Technology Projects in Jingmen City (No.
YFYB2017006).

Conflict of Interest The authors declare no conflict of interest.

\section{References}

1) Fattahi F., Russell M. W., Malan E. A., Parlett M., Abe E., Zetoune F. S., Ward P. A., BioMed Research International, 2018, 4302726 (2018).

2) Pavon A., Binquet C., Kara F., Martinet O., Ganster F., Navellou J. C., Castelain V., Barraud D., Cousson J., Louis G., Perez P., Kuteifan K., Noirot A., Badie J., Mezher C., Lessire H., Quantin C. Abrahamowicz M., Quenot J. P., Crit. Care Med., 41, 2600-2609 (2013).

3) Zarjou A., Black L. M., McCullough K. R., Hull T. D., Esman S. K., Boddu R., Varambally S., Chandrashekar D. S., Feng W., Arosio P., Poli M., Balla J., Bolisetty S., Front. Immunol., 10, 131 (2019).

4) Peng X., Ding Y., Wihl D., Gottesman O., Komorowski M., Lehman L. H., Ross A., Faisal A., Doshi-Velez F., AMIA Annual Symposium proceedings AMIA Symposium, 2018, 887-896 (2018).

5) Makara M. A., Hoang K. V., Ganesan L. P., Crouser E. D., Gunn J. S., Turner J., Schlesinger L. S., Mohler P. J., Rajaram M. V., Journal of the American Heart Association., 5, e003820 (2016).

6) Corrales-Medina V. F., Mushor D. M., Wells G. A., Chirinos J. A., Chen L., Fine M. J., Circulation, 125, 773-781 (2012).

7) Zhou M. Q., Shao L., Wu J., Peng N., Jin L. P., Wei G. Z., Cheng W., Deng C. J., Mol Med Rep., 16, 8983-8988 (2017).

8) Vieillard-Baron A., Caille V., Charron C., Belliard G., Page B., Jardin F., Crit. Care Med., 36, 1701-1706 (2008).

9) Parrillo J. E., Parker M. M., Natanson C., Suffredini A. F., Danner R. L., Cunnion R. E., Ognibene F. P., Ann. Intern. Med., 113, 227-242 (1990)

10) Bai B., Ban B., Liu Z., Zhang M. M., Tan B. K., Chen J., PLOS ONE, 12, e0172271 (2017).

11) Enomoto T., Ohashi K., Shibata R., Higuchi A., Maruyama S., Izumiya Y., Walsh K., Murohara T., Ouchi N., J. Biol. Chem., 286, 34552-34558 (2011)

12) Tan S. Y., Little H. C., Lei X., Li S., Rodriguez S., Wong G. W., Physiol. Genomics, 48, 936-949 (2016).

13) Fadaei R., Moradi N., Kazemi T., Chamani E., Azdaki N., Moezibady S. A., Shahmohamadnejad S., Fallah S., Cytokine, 113, 326-331 (2019).

14) Liu Y., Hu Z. F., Liao H. H., Liu W., Liu J., Ma Z. G., Wu Q. Q., Xu M., Zhang N., Zhang Y., Bian Z. Y., Tang Q. Z., Biochim. Biophys. Acta, 1852, 2456-2466 (2015).

15) Liu Y. C., Yu M. M., Shou S. T., Chai Y. F., Front. Immunol., 8, $1021(2017)$

16) Suffredini A. F., Fromm R. E., Parker M. M., Brenner M., Kovacs J. A., Wesley R. A., Parrillo J. E., N. Engl. J. Med., 321, 280-287 (1989)

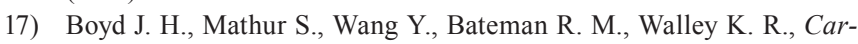
diovasc. Res., 72, 384-393 (2006).

18) Eichenholz P. W., Eichacker P. Q., Hoffman W. D., Banks S. M., Parrillo J. E., Danner R. L., Natanson C., Am. J. Physiol., 263, H668-H675 (1992).

19) Tsolaki V., Makris D., Mantzarlis K., Zakynthinos E., Oxid. Med. Cell. Longev., 2017, 7393525 (2017).

20) Zimmet J. M., Hare J. M., Circulation, 114, 1531-1544 (2006).

21) Sheeran F. L., Pepe S., Adv. Exp. Med. Biol., 982, 65-80 (2017).

22) Smith R. E., Tran K., Smith C. C., McDonald M., Shejwalkar P., Hara K., Diseases, 4, 34 (2016).

23) Chen Q. M. M. A., Maltagliati A. J., Physiol. Genomics, 50, 77-97 (2018). 